\title{
Successful treatment of non-metastatic hepatoblastoma in patient with trisomy 18 using modified chemotherapy regimen
}

\author{
Stephanie Schmitt ${ }^{1}$ and Erin Trovillion ${ }^{2}$ \\ ${ }^{1}$ Rady Children's Hospital San Diego \\ ${ }^{2}$ Atrium Health
}

December 2, 2020

\begin{abstract}
To date, there have only been 46 patients reported in the literature with trisomy 18 and hepatoblastoma. We describe the case of an 11-month-old female with trisomy 18 and hepatoblastoma who was successfully treated by pairing surgical resection with a modified chemotherapy regimen omitting doxorubicin given her underlying cardiac abnormalities. Our case successfully demonstrates how chemotherapy regimens can be modified to accommodate for other underlying abnormalities and emphasizes that routine screening guidelines should exist for patients with trisomy 18 to allow for earlier detection and treatment.
\end{abstract}

Successful treatment of non-metastatic hepatoblastoma in patient with trisomy 18 using modified chemotherapy regimen

Stephanie Schmitt $\mathrm{MD}^{1}$; Erin Murphy Trovillion $\mathrm{MD}^{2}$

${ }^{1}$ University of California San Diego, Department of Pediatrics

${ }^{2}$ Atrium Health Medical Group Division, Levine Children's Cancer and Blood Disorders

Keywords: Hepatoblastoma, Trisomy 18, Pediatric, Oncology

Corresponding Author:

Erin M. Trovillion, MD

1001 Blythe Boulevard, Suite 601, Charlotte, NC 28203

Phone: 704-381-9902; Fax: 704-381-9901

Text Word Count: 1000

Abstract Word Count: 86

Number of figures: 1

Short Running Title: treatment of hepatoblastoma in patient with Trisomy 18

Abbreviations Table

\begin{tabular}{ll}
\hline AFP & Alpha-fetoprotein \\
\hline $\mathrm{Cm}$ & Centimeter \\
$\mathrm{COG}$ & Children's Oncology Group \\
$\mathrm{CT}$ & Computed Tomography \\
ICU & Intensive Care Unit
\end{tabular}




\begin{tabular}{ll}
\hline AFP & Alpha-fetoprotein \\
\hline US & Ultrasound \\
\hline
\end{tabular}

\section{ABSTRACT:}

To date, there have only been 46 patients reported in the literature with trisomy 18 and hepatoblastoma. We describe the case of an 11-month-old female with trisomy 18 and hepatoblastoma who was successfully treated by pairing surgical resection with a modified chemotherapy regimen omitting doxorubicin given her underlying cardiac abnormalities. Our case successfully demonstrates how chemotherapy regimens can be modified to accommodate for other underlying abnormalities and emphasizes that routine screening guidelines should exist for patients with trisomy 18 to allow for earlier detection and treatment.

\section{INTRODUCTION:}

Trisomy 18, also known as Edwards syndrome, is the second most common congenital trisomy genetic disorder after Down's Syndrome. It is associated with multiple, often devastating, cardiac and pulmonary anomalies, other structural malformations, and developmental delays. ${ }^{1}$ Though Trisomy 18 is still frequently described as "incompatible with life," studies have shown that if an infant with Trisomy 18 survives beyond the first weeks after birth where mortality nears $50 \%$, that over $10 \%$ of children will survive the first year of life. ${ }^{2,3}$ With evidence demonstrating that medical and surgical interventions can further improve survival and outcomes and our ever-evolving medical capabilities, these children are expected to survive even longer still. $^{2}$

Recently, there have been increasing reports of hepatoblastoma in individuals with Trisomy 18. As of 2019, there have been 46 total described cases of patients with trisomy 18 and hepatoblastoma, raising concerns about an association between the two. ${ }^{1,4}$ Despite the controversies that persist surrounding aggressive interventions in patients with Trisomy 18, several patients with hepatoblastoma, including our patient have been successfully treated with chemotherapy and/or surgery resection. ${ }^{1,4}$ This report describes a patient with Trisomy 18 and Stage III, intermediate risk hepatoblastoma successfully treated with neoadjuvant chemotherapy and subsequent resection without the use of doxorubicin.

\section{CASE DESCRIPTION:}

We describe the case of an 11-month-old female diagnosed with trisomy 18 and hepatoblastoma. She was born at 37 weeks gestation via cesarean section due to concern for placental insufficiency and intrauterine growth restriction. In the neonatal intensive care unit (ICU), she was found to have persistent pulmonary hypertension, a small ventricular septal defect, a moderate atrial septal defect, pulmonic stenosis and a large patent ductus arteriosus. She was also found to have congenital hydronephrosis with duplicated collecting system and imperforate anus with rectovaginal fistulae. An underlying genetic condition was strongly suspected and the diagnosis of trisomy 18 was confirmed by fluorescence in situ hybridization.

At 8 months of age she was hospitalized for two months in India and England, requiring ICU level of care for bacteremia, sepsis, and respiratory failure. Given recurrence of feeding difficulties in the context of prolonged hospitalization and multiple intubations, multiple abdominal ultrasounds (US) were performed. Her third abdominal US revealed a liver mass that measured $3.7 \mathrm{~cm} \times 3.6 \mathrm{~cm} \times 3.3 \mathrm{~cm}$. Parents were told that based on imaging, this lesion was most likely benign, specifically a hemangioma. Computed tomography (CT) and serum $\alpha$-fetoprotein level (AFP) were recommended but given severity of illness, further evaluation was deferred.

Three months later, upon family's return to the United States, a repeat US was performed which demonstrated a prominent mass concerning for malignancy that replaced the majority of the left lower liver measuring $8.9 \mathrm{~cm} \times 4.5 \mathrm{~cm} \times 6.1 \mathrm{~cm}$. CT guided biopsy of the liver mass was performed re-demonstrating a large, exophytic hypervascular mass in the left lobe of the liver. CT chest was without distant metastatic disease. Given proximity to the middle hepatic vein, surgical resection was not possible at time of diagnosis. 
Pathology was consistent with pure fetal histology hepatoblastoma. Despite favorable pathology, given unresectable tumor, patient was diagnosed with Stage III, intermediate risk disease. AFP at diagnosis was $17,657 \mathrm{ng} / \mathrm{mL}$ (Figure 1).

The patient completed neo-adjuvant chemotherapy as per the Children's Oncology Group (COG) protocol AHEP0731 (Stratum 3, Regimen F) for two cycles using cisplatin, fluorouracil, and vincristine. After consultation with the cardiology team, doxorubicin was omitted from her treatment given her underlying cardiac abnormalities. The patient successfully underwent a left lateral hepatic sectionectomy with clean margins after two cycles of chemotherapy. After surgical resection, she received one adjuvant cycle of chemotherapy as per AHEP0731. After discussion with family, given clear surgical margins and baseline clinical fragility opted to discontinue chemotherapy after cycle 3 . At her visit 3 months from the end of therapy, patient continues to do well with normal AFP level and CT chest and magnetic resonance imaging of abdomen/pelvis are without evidence of recurrent, residual or metastatic disease (Figure 1).

\section{DISCUSSION:}

The extent of both screening and care for children with trisomy 18 is a complex, individualized and subjective discussion, unique to each patient and family. However, this emerging association between hepatoblastoma and trisomy 18, with preliminary studies estimating $>1 \%$ risk of hepatoblastoma, raises questions about the potential benefits of routine screening to promote earlier diagnosis and minimize need for neoadjuvant chemotherapy. Children with Beckwith Weideman syndrome and WT1 -related overgrowth syndromes have a known predisposition for both hepatoblastoma and Wilms tumor. Tumor surveillance consisting of interval abdominal US (every 3 months until age 7) and serial AFP measurement (a baseline at birth then every 3 months until age 4) are recommended given the proven advantages of early diagnosis in prognosis. ${ }^{5}$ As AFP is a highly specific and sensitive tumor marker, secreted by over $96 \%$ of hepatoblastomas, it serves as an effective screening tool in at risk patients, as it can rise prior to tumor detection with US or other imaging modalities. ${ }^{6,7}$ In 2019 results of a literature search were released confirming a high number of reported malignancies in Trisomy 18 patients. Based on this, initial screening recommendations for abdominal malignancies were published, however this has not yet become the standard of care. ${ }^{1}$

All COG study protocols include dose modification guidelines in the event that patients experience toxicities related to chemotherapy. In AHEP0731, omission of doxorubicin is recommended in Stage III, IntermediateRisk Hepatoblastoma only if the patient develops persistent grade 3 or 4 mucositis despite dose reduction, or for Grade 3 congestive heart failure, Grade 4 cardiac toxicity or major changes in cardiac ejection fractions attributable to doxorubicin. ${ }^{8}$ Despite an initially unresectable tumor, our patient with trisomy 18 and extensive cardiac history was effectively treated for intermediate risk hepatoblastoma with modified chemotherapy without doxorubicin followed by surgical resection. This supports offering patients with Trisomy 18 and underlying cardiac abnormalities modified chemotherapy regimens as curative treatment for hepatoblastoma. Regardless, the care of individuals with trisomy 18 is not homogeneous. It should instead be family-centered, flexible, and reflect parents' views that their children with trisomy 18 are "happy" and "enriching to their lives." 9

\section{CONFLICT OF INTEREST STATEMENT:}

Authors do not have any conflicts of interest.

\section{ACKNOWLEDGEMENTS:}

Consent was provided by the patient's family to publish information regarding her medical condition and treatment.

\section{CITATIONS:}

1. Farmakis SG, Barnes AM, Carey JC, Braddock SR. Solid tumor screening recommendations in trisomy 18. Am J Med Genet A . 2019;179(3):455-466. doi:10.1002/ajmg.a.61029 
2. Silberberg A, Robetto J, Grimaux G, Nucifora L, Moreno Villares JM. Ethical issues about the paradigm shift in the treatment of children with trisomy 18. Eur J Pediatr . 2020;179(3):493-497. doi:10.1007/s00431019-03531-4

3. Goel N, Morris JK, Tucker D, et al. Trisomy 13 and 18-Prevalence and mortality-A multi-registry population based analysis. Am J Med Genet A . 2019;179(12):2382-2392. doi:10.1002/ajmg.a.61365

4. Lucas DJ, Rubinstein J, Gosain A, et al. Surgical and anesthetic management for hepatectomy in two pediatric patients with trisomy 18, pulmonary hypertension, and hepatoblastoma. Pediatr Blood Cancer . 2019;66(6):e27678. doi:10.1002/pbc.27678

5. Kalish JM, Doros L, Helman LJ, et al. Surveillance Recommendations for Children with Overgrowth Syndromes and Predisposition to Wilms Tumors and Hepatoblastoma. Clin Cancer Res Off J Am Assoc Cancer Res . 2017;23(13):e115-e122. doi:10.1158/1078-0432.CCR-17-0710

6. Clericuzio CL, Chen E, McNeil DE, et al. Serum $\alpha$-fetoprotein screening for hepatoblastoma in children with Beckwith-Wiedemann syndrome or isolated hemihyperplasia. J Pediatr . 2003;143(2):270-272. doi:10.1067/S0022-3476(03)00306-8

7. Zarate YA, Mena R, Martin LJ, Steele P, Tinkle BT, Hopkin RJ. Experience with hemihyperplasia and Beckwith-Wiedemann syndrome surveillance protocol. Am J Med Genet A . 2009;149A(8):1691-1697. doi:10.1002/ajmg.a.32966

8. Children's Oncology Group AHEP0731 Treatment of Children with All Stages of

Hepatoblastoma with Temsirolimus (IND\#122782, NSC\#683664) Added to High

Risk Stratum Treatment, A Phase III Study. Version Date: 11/07/18.

9. Janvier A, Farlow B, Wilfond BS. The Experience of Families With Children With Trisomy 13 and 18 in Social Networks. Pediatrics . 2012;130(2):293-298. doi:10.1542/peds.2012-0151

FIGURE LEGEND:

Figure 1: Graph depicting fall in AFP following chemotherapy and resection. Patient continues to have normal AFP levels 3 months from end of therapy. Reference Range: $0.5-11.1 \mathrm{ng} / \mathrm{mL}$. Abbreviations: PostResection (PR), End of Treatment (EOT). 


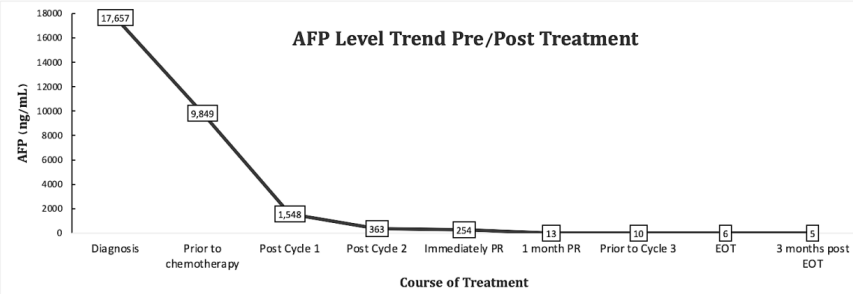

FIGURE 1: Graph depicting fall in AFP following chemotherapy and resection. Patient continues to have normal AFP levels 3 months from end of therapy. Reference Range: $0.5-11.1 \mathrm{ng} / \mathrm{mL}$. Abbreviations: PostResection (PR), End of Treatment (EOT). 